\title{
Downlink Linear Transmission Schemes in a Single-Cell Distributed Antenna System with Port Selection
}

\author{
Talha Ahmad*, Saad Al-Ahmadi ${ }^{\dagger}$, Halim Yanikomeroglu*, Gary Boudreau ${ }^{\ddagger}$ \\ * Department of Systems and Computer Engineering, Carleton University, Ottawa, Ontario, Canada \\ $\dagger$ Electrical Engineering Department, King Fahd University of Petroleum \& Minerals, Dhahran, Saudi Arabia \\ $\ddagger$ Ericsson Canada, Ottawa, Ontario, Canada \\ Email: \{tahmad, halim\}@sce.carleton.ca, ahmadisa@kfupm.edu.sa, gary.boudreau@ericsson.com
}

\begin{abstract}
A cellular distributed antenna system (DAS) allows mobile user terminals (UTs) to be served at higher data rates as compared to conventional cellular systems by reducing the path loss and attaining macrodiversity gains. Moreover, such a cellular DAS can be perceived as a distributed multi-user multiple-input multiple-output system. In this paper, the block diagonalization and zero-forcing dirty-paper coding downlink transmission schemes are extended for a single-cell DAS with multi-antenna distributed antenna ports (DAPs) and multi-antenna UTs, where only a subset of all DAPs in the cell transmit to each UT. The aggregate cell spectral efficiency that is achieved by these schemes per frequency-time resource block is compared for both DAS and collocated antenna system (CAS) architectures, subject to the same total power constraint. The gains of the cellular DAS over the cellular CAS are demonstrated, and the effect of the number of antennas per DAP on the performance of the cellular DAS is investigated.
\end{abstract}

Index Terms-Distributed antenna system, coordinated transmission, multi-user MIMO, block diagonalization, zero-forcing dirty-paper coding.

\section{INTRODUCTION}

Future cellular networks are expected to provide high data rate service throughout the cell. In conventional cellular networks, a user terminal (UT) nearby the base station (BS) can be served at a relatively high data rate. However, UTs that are further away from the BS experience significant path loss, which leads to low data rates. A cellular distributed antenna system (DAS) is a promising candidate architecture that can help in enabling ubiquitous coverage over the entire cell. In a cellular DAS, several antenna ports are geographically separated from each other, and each distributed antenna port (DAP) is connected to the BS via a fiber optic link or another dedicated wired medium.

Distributed antenna systems were first introduced in [1] to improve the coverage distance for mobile UTs in an indoor environment, and early research on DAS was in this context [2]. Early works on the integration of DAS in cellular networks appeared in [3]-[7]. In [3], the uplink outage probability of a generalized DAS was evaluated for various diversity combining schemes using a composite fading channel model. In [4], a code division multiple access (CDMA) sectorized distributed antenna architecture was proposed. Outage capacity analysis of a CDMA DAS was performed for both uplink and downlink in [5], and the outage capacity of multiple-input multiple-output (MIMO) DAS was studied in [6], [7].

In comparison to the collocated antenna system (CAS), i.e., a conventional cellular system, deploying a DAS leads to reduced path loss from certain DAPs to a particular UT, as well as independent shadowing statistics from/to each DAP. Additionally, all antennas at the BS are usually used to transmit to each UT in a CAS, whereas in the case of a DAS, the distance from certain DAPs may be too large to spend power for transmission to particular UTs. Subsets of the available DAPs can, instead, be used to transmit to individual UTs [8]. As a result, it may not be necessary for some DAPs in the cell to be active if no UTs are nearby.

A single-cell CAS with multi-antenna UTs can be modeled as a Gaussian MIMO broadcast channel (BC), for which the optimal transmission scheme is dirty-paper coding (DPC) [9]. However, DPC is difficult to implement in practice due to its computational complexity, and several sub-optimal linear and non-linear transmission schemes have been proposed for mitigating inter-user interference [10]-[16]. In [10], the zeroforcing dirty-paper coding (ZF-DPC) scheme is proposed, which performs LQ decomposition on the aggregate channel matrix consisting of the channel matrices of all single-antenna UTs, to eliminate a part of the inter-user interference. The remaining interference is mitigated by means of successive dirtypaper encoding. ZF-DPC is shown in [10] to be asymptotically optimal with increasing signal-to-noise ratio (SNR). ZF-DPC has been extended to incorporate multi-antenna UTs in [11][14]. A linear scheme that mitigates inter-user interference is zero-forcing beamforming (ZFBF), which orthogonalizes all single-antenna UTs using the pseudo-inverse of the aggregate channel matrix. An extension of this scheme for multi-antenna UTs is block diagonalization (BD) [15], in which each UT's precoding matrix is designed such that its transmitted signal lies in the null space of the channel matrices of the other UTs.

With the incorporation of port selection, the CAS-based versions of these schemes cannot be directly applied to a DAS, and processing modifications are necessary. In this paper, the 
ZF-DPC and BD schemes are extended to fit the cellular DAS architecture. In [17], the performance of various multi-user transmission schemes, including BD, is explored in the context of a single-cell DAS with multi-antenna DAPs. However, the schemes presented in [17] do not incorporate port selection. Furthermore, single-antenna UTs are mainly assumed and spatial multiplexing of multiple data streams for each UT is not included. Both these features are considered in this paper. In [18], port selection is explored for a multi-user cellular DAS with the selection criteria being based on path loss alone, as well as a combination of path loss and multipath fading. However, the UTs are orthogonalized through orthogonal frequency division multiplexing (OFDM), whereas orthogonalization is achieved through spatial precoding in this paper.

\section{Signal And Channel Models}

Consider a single-cell DAS with $D M$-antenna DAPs, including one at the BS itself. All DAPs are located uniformly throughout the cell, and are connected to the BS by a lossless and delayless link. There is a central processor at the BS, which has perfect knowledge of the instantaneous channel gains between each active DAP and each UT in the network, i.e., perfect channel state information at the transmitter (CSIT). There are $K N$-antenna UTs in the cell. Such a cellular DAS is illustrated in Fig. 1. Let $\mathcal{S}$ be the set of indices of all DAPs in the system, where $|\mathcal{S}|=D$ and $|\cdot|$ denotes the cardinality of the enclosed set. Let $\mathcal{A}$ represent the set of all active DAPs $(\mathcal{A} \subseteq \mathcal{S})$. Also, let $\mathcal{C}_{k}$ and $\mathcal{I}_{k}$ represent the set of DAPs that cooperatively transmit to the $k^{t h}$ UT and the set of DAPs that cause interference to that UT, respectively.

\section{A. Signal Model}

The received signal at the $k^{t h} \mathrm{UT}$ can be expressed as

$$
\boldsymbol{y}_{k}=\boldsymbol{H}_{k} \boldsymbol{x}+\boldsymbol{n}_{k}, k=1, \ldots, K,
$$

where $\boldsymbol{y}_{k} \in \mathbb{C}^{N \times 1}, \boldsymbol{H}_{k} \in \mathbb{C}^{N \times|\mathcal{A}| M}$ is the channel transfer matrix between the $k^{t h}$ UT and all active DAPs in the cell, containing path loss, shadowing, as well as multipath fading, $\boldsymbol{n}_{k} \in \mathbb{C}^{N \times 1}$ is a zero-mean complex Gaussian noise vector with covariance matrix $E\left[\boldsymbol{n}_{k} \boldsymbol{n}_{k}^{H}\right]=\sigma_{n}^{2} \boldsymbol{I}_{N}$, and $\boldsymbol{x} \in \mathbb{C}^{|\mathcal{A}| M \times 1}$ is the transmitted signal defined as

$$
\boldsymbol{x}=\sum_{k=1}^{K} \boldsymbol{F}_{k}^{\prime} \boldsymbol{d}_{k}
$$

where $\boldsymbol{d}_{k} \in \mathbb{C}^{N \times 1}$ is the data vector of the $k^{t h} \mathrm{UT}$, and $\boldsymbol{F}_{k}^{\prime} \in \mathbb{C}^{|\mathcal{A}| M \times N}$ is its precoding matrix. This precoding matrix is of the form $\boldsymbol{F}_{k}^{\prime}=\boldsymbol{F}_{k} \boldsymbol{\Lambda}_{k}^{\frac{1}{2}}$, where $\boldsymbol{F}_{k} \in \mathbb{C}^{|\mathcal{A}| M \times N}$ is the transmit beamforming matrix and $\boldsymbol{\Lambda}_{k} \in \mathbb{C}^{N \times N}$ is a diagonal power allocation matrix. Eq. (1) can then be re-written as

$$
\boldsymbol{y}_{k}=\boldsymbol{H}_{k} \boldsymbol{F}_{k} \boldsymbol{\Lambda}_{k}^{\frac{1}{2}} \boldsymbol{d}_{k}+\boldsymbol{H}_{k} \sum_{i=1, i \neq k}^{K} \boldsymbol{F}_{i} \boldsymbol{\Lambda}_{i}^{\frac{1}{2}} \boldsymbol{d}_{i}+\boldsymbol{n}_{k},
$$

where the second term on the right-hand side of (3) represents the inter-user interference experienced by the $k^{t h} \mathrm{UT}$.
The cellular DAS is subject to a total power constraint, $E\left[\boldsymbol{x} \boldsymbol{x}^{H}\right] \leq P_{t}$, where $P_{t}$ is the total available transmit power. Assuming that the data vectors, $\boldsymbol{d}_{k}, \forall k=1, \ldots, K$, are zeromean with identity covariance matrix, this constraint can be written as $\sum_{k=1}^{K} \operatorname{Tr}\left(\boldsymbol{S}_{k}\right) \leq P_{t}$, where $\boldsymbol{S}_{k}=\boldsymbol{F}_{k}^{\prime} \boldsymbol{F}_{k}^{\prime H}$ is the transmit covariance matrix of the $k^{\text {th }} \mathrm{UT}$ [19], and $\operatorname{Tr}(\cdot)$ is the trace operator.

It is worth noting that a per-DAP power constraint is more practical; however, we consider the total power constraint to enable a fair comparison between the performance of the DAS and of an equivalent CAS.

\section{B. Channel Model}

The channel matrix $\boldsymbol{H}_{k}$ in (1) for the DAS is of the form

$$
\boldsymbol{H}_{k}=\left[\begin{array}{llll}
\boldsymbol{H}_{k}^{(1)} & \boldsymbol{H}_{k}^{(2)} & \ldots & \boldsymbol{H}_{k}^{(|\mathcal{A}|)}
\end{array}\right], k=1, \ldots, K,
$$

where $\boldsymbol{H}_{k}^{(i)}=\sqrt{s_{k}^{(i)}\left(\frac{d_{k}^{(i)}}{d_{k}^{(B S)}}\right)^{-\beta}} \boldsymbol{H}_{k}^{(i)^{\prime}}, d_{k}^{(i)}$ is the distance between the $k^{t h} \mathrm{UT}$ and the $i^{t h} \mathrm{DAP}, d_{k}^{(B S)}$ is the distance between this UT and the BS, i.e., the cell center, $\beta$ is the path loss exponent, $\boldsymbol{H}_{k}^{(i)^{\prime}} \in \mathbb{C}^{N \times M}$ contains the multipath fading coefficients, and $s_{k}^{(i)}$ is the shadowing coefficient, which is assumed to have a gamma distribution with shape parameter $m_{s}$ and scale parameter $\frac{\Omega_{s}}{m_{s}}$, where $\Omega_{s}$ is the mean power in the cell area. The gamma distribution approximates the lognormal distribution, which is conventionally used to model shadowing in wireless channels. This approximation is generally adopted due to the analytical simplicity of the gamma distribution [20].

In the case of a CAS, the channel matrix can be written as

$$
\boldsymbol{H}_{k}=\sqrt{s_{k}} \boldsymbol{H}_{k}^{\prime}
$$

where $s_{k}$ is the shadowing coefficient between the $k^{\text {th }} \mathrm{UT}$ and $|\mathcal{A}| M$ antennas collocated at the BS, and $\boldsymbol{H}_{k}^{\prime} \in \mathbb{C}^{N \times|\mathcal{A}| M}$ is the multipath fading coefficient matrix. The path loss component is set to unity due to the normalization by $d_{k}^{(B S)}$.

\section{Proposed DOWNLINK TRANSMISSION SCHEMES}

\section{A. DAS Block Diagonalization}

Considering the first term on the right-hand side of (3), the rows of $\boldsymbol{F}_{k}$ corresponding to those columns of $\boldsymbol{H}_{k}$ that represent the transmit antennas of the DAPs in $\mathcal{I}_{k}$, must be set to zero, since those particular DAPs do not transmit desired signals to the $k^{t h}$ UT. The remaining non-zero rows of $\boldsymbol{F}_{k}$ then constitute the submatrix $\hat{\boldsymbol{F}}_{k} \in \mathbb{C}^{\left|\mathcal{C}_{k}\right| M \times N}$.

Eliminating inter-user interference requires the following condition to be satisfied: $\boldsymbol{H}_{i} \boldsymbol{F}_{k}=0, \forall i \neq k$, which can be reduced to $\hat{\boldsymbol{H}}_{i} \hat{\boldsymbol{F}}_{k}=0, \forall i \neq k$, where $\hat{\boldsymbol{H}}_{k} \in \mathbb{C}^{N \times\left|\mathcal{C}_{k}\right| M}$ is a channel matrix containing only those columns of $\boldsymbol{H}_{k}$ that correspond to the transmit antennas of the DAPs in $\mathcal{C}_{k}$. The interference matrix of the $k^{t h} \mathrm{UT}$ is defined as

$$
\begin{aligned}
& \tilde{\boldsymbol{H}}_{k}=\left[\begin{array}{llllll}
\overline{\boldsymbol{H}}_{1, k}^{H} & \ldots & \overline{\boldsymbol{H}}_{k-1, k}^{H} & \overline{\boldsymbol{H}}_{k+1, k}^{H} & \ldots & \overline{\boldsymbol{H}}_{K, k}^{H}
\end{array}\right]^{H}, \\
& k=1, \ldots, K
\end{aligned}
$$


where $\overline{\boldsymbol{H}}_{i, k} \in \mathbb{C}^{N \times\left|\mathcal{C}_{k}\right| M}$ is a submatrix containing only those columns of $\boldsymbol{H}_{i}$ that correspond to the transmit antennas of the DAPs in $\mathcal{C}_{k}$. Assuming that $\tilde{\boldsymbol{H}}_{k}$ is full-rank, let $\tilde{L}_{k}=\operatorname{rank}\left(\tilde{\boldsymbol{H}}_{k}\right)=\min \left\{N(K-1),\left|\mathcal{C}_{k}\right| M\right\}$. The singular value decomposition (SVD) of $\tilde{\boldsymbol{H}}_{k}$ is given by

$$
\tilde{\boldsymbol{H}}_{k}=\tilde{\boldsymbol{U}}_{k} \tilde{\boldsymbol{\Sigma}}_{k}\left[\begin{array}{ll}
\tilde{\boldsymbol{V}}_{k}^{(1)} & \tilde{\boldsymbol{V}}_{k}^{(0)}
\end{array}\right]^{H},
$$

where $\tilde{\boldsymbol{V}}_{k}^{(1)}$ contains in its columns the first $\tilde{L}_{k}$ right singular vectors of $\tilde{\boldsymbol{H}}_{k}$, and $\tilde{\boldsymbol{V}}_{k}^{(0)}$ contains the remaining $\left|\mathcal{C}_{k}\right| M-\tilde{L}_{k}$ right singular vectors. In order for $\tilde{\boldsymbol{V}}_{k}^{(0)}$ to be non-empty for all $k$, the condition,

$$
N(K-1)<\min _{k \in\{1, \ldots, K\}}\left(\left|\mathcal{C}_{k}\right| M\right),
$$

must be satisfied. This is the dimensionality constraint of a cellular DAS with port selection, which limits the number of UTs that can be simultaneously served in a particular time and frequency slot, which is hereby referred to as a resource block $(\mathrm{RB})^{1}$. The columns of $\tilde{\boldsymbol{V}}_{k}^{(0)}$ then form an orthogonal basis for the null space of $\tilde{\boldsymbol{H}}_{k} . \hat{\boldsymbol{F}}_{k}$ can, therefore, be designed by using a linear combination of the columns of $\tilde{\boldsymbol{V}}_{k}^{(0)}$, such that the effective channel gain is maximized. This is achieved by performing the following SVD [15],

$$
\hat{\boldsymbol{H}}_{k} \tilde{\boldsymbol{V}}_{k}^{(0)}=\hat{\boldsymbol{U}}_{k}\left[\begin{array}{cc}
\hat{\boldsymbol{\Sigma}}_{k} & \mathbf{0} \\
\mathbf{0} & \mathbf{0}
\end{array}\right]\left[\begin{array}{ll}
\hat{\boldsymbol{V}}_{k}^{(1)} & \hat{\boldsymbol{V}}_{k}^{(0)}
\end{array}\right]^{H}
$$

where $\hat{\boldsymbol{\Sigma}}_{k} \in \mathbb{C}^{\bar{L}_{k} \times \bar{L}_{k}}$ is a diagonal matrix comprised of the singular values of $\hat{\boldsymbol{H}}_{k} \tilde{\boldsymbol{V}}_{k}^{(0)}$ as its diagonal elements, $\bar{L}_{k}=$ $\operatorname{rank}\left(\hat{\boldsymbol{H}}_{k} \tilde{\boldsymbol{V}}_{k}^{(0)}\right)$, and $\hat{\boldsymbol{V}}_{k}^{(1)}$ contains the first $N$ right singular vectors of $\hat{\boldsymbol{H}}_{k} \tilde{\boldsymbol{V}}_{k}^{(0)}$. Then, $\hat{\boldsymbol{F}}_{k}$ is defined as

$$
\hat{\boldsymbol{F}}_{k}=\tilde{\boldsymbol{V}}_{k}^{(0)} \hat{\boldsymbol{V}}_{k}^{(1)} \text {. }
$$

Note that this transmit beamforming matrix is designed to only mitigate inter-user interference. The task of separating multiple data streams per UT is left for the processing at each UT itself, since complete orthogonalization of all data streams at the transmitter is suboptimal in terms of maximum achievable aggregate cell spectral efficiency [15]. Each UT can separate its own data streams by using a decoding matrix of $\hat{\boldsymbol{U}}_{k}^{H}$, where $\hat{U}_{k}$ is obtained from (9). The optimal power allocation matrix, $\boldsymbol{\Lambda}=\operatorname{diag}\left(\boldsymbol{\Lambda}_{1}, \ldots, \boldsymbol{\Lambda}_{K}\right)$, is obtained by performing waterfilling on the diagonal elements of $\hat{\boldsymbol{\Sigma}}=\operatorname{diag}\left(\hat{\boldsymbol{\Sigma}}_{1}, \ldots, \hat{\boldsymbol{\Sigma}}_{K}\right)$ under the total transmit power constraint $P_{t}$. The aggregate cell spectral efficiency per RB is given by

$$
\begin{aligned}
R_{\text {cell }, B D} & =\sum_{k=1}^{K} \log _{2} \operatorname{det}\left(\boldsymbol{I}_{N}+\frac{\left(\hat{\boldsymbol{U}}_{k}^{H} \boldsymbol{H}_{k} \boldsymbol{F}_{k} \boldsymbol{\Lambda}_{k}^{\frac{1}{2}}\right)^{2}}{\sigma_{n}^{2}}\right) \\
& =\sum_{k=1}^{K} \log _{2} \operatorname{det}\left(\boldsymbol{I}_{N}+\frac{\hat{\boldsymbol{\Sigma}}_{k}^{2} \boldsymbol{\Lambda}_{k}}{\sigma_{n}^{2}}\right)
\end{aligned}
$$

\footnotetext{
${ }^{1}$ The DAS dimensionality constraint does not, however, impose a strict limit on the overall number of active UTs since a different set of UTs can be selected for service in another RB using an appropriate scheduling algorithm.
}

\section{B. DAS Zero-Forcing Dirty-Paper Coding}

The formulation in this section is an extension of the ZFDPC scheme described in [12] for multi-antenna UTs. Let $\pi$ represent a permutation operator for UT ordering. Successive dirty-paper encoding is performed to generate the $\pi(k)^{t h}$ UT's data vector $\boldsymbol{d}_{\pi(k)}$, such that interference from all UTs that have a lower index is eliminated, while the remaining interference is mitigated by means of BD-based zero-forcing.

Similar to the DAS BD scheme, the interference matrix of the $\pi(k)^{t h}$ UT is now defined as

$$
\begin{aligned}
& \tilde{\boldsymbol{H}}_{\pi(k)}=\left[\begin{array}{llll}
\overline{\boldsymbol{H}}_{\pi(1), \pi(k)}^{H} & \overline{\boldsymbol{H}}_{\pi(2), \pi(k)}^{H} & \ldots & \overline{\boldsymbol{H}}_{\pi(k-1), \pi(k)}^{H}
\end{array}\right]^{H}, \\
& k=2, \ldots, K \text {. }
\end{aligned}
$$

The transmit beamforming matrix design and power allocation from this point onward are identical to those described for the DAS BD scheme. Note that no interference matrix exists for the $\pi(1)^{s t}$ UT since the beamforming matrices of all other UTs are designed to mitigate interference to this UT. In this case, SVD is applied directly to $\hat{\boldsymbol{H}}_{\pi(1)}$, and the resulting matrix $\hat{\boldsymbol{\Sigma}}_{\pi(1)}$ is used for power allocation. The maximum achievable aggregate cell spectral efficiency per RB is given by

$$
R_{\text {cell }, Z F-D P C}=\max _{\pi} \sum_{k=1}^{K} \log _{2} \operatorname{det}\left(\boldsymbol{I}_{N}+\frac{\hat{\boldsymbol{\Sigma}}_{\pi(k)}^{2} \boldsymbol{\Lambda}_{\pi(k)}}{\sigma_{n}^{2}}\right)_{(13)}
$$

where the maximization over $\pi$ is due to the fact that UT ordering affects the achievable spectral efficiency. For simplicity, however, we consider an arbitrary UT ordering in this paper.

\section{Performance Evaluation}

The performance of the cellular DAS transmission schemes is evaluated through Monte Carlo simulations, and is compared with that of the equivalent schemes in a corresponding cellular CAS. The same total power constraint is applied to both architectures to enable fair comparison. As well, the number of active antennas that transmit to each UT in the DAS, is preserved in the case of the CAS by selecting those $\left|\mathcal{C}_{k}\right| M$ antennas out of the total $|\mathcal{A}| M$ available antennas in the CAS that have the largest channel gains (including both large-scale and small-scale fading) to the $k^{\text {th }}$ UT.

Port selection can be viewed as a special case of transmit antenna selection. With antenna selection, a subset of all transmit antennas with the largest channel gains to a particular UT are selected to transmit to it, whereas with port selection, antennas at each DAP are selected as a group. We explicitly point out that unlike antenna selection, the criteria used for port selection in our simulations is large-scale fading (path loss and shadowing) only. The performance of both approaches is compared in this section and it is shown that although port selection is less flexible, the performance gap between the two approaches is negligible. Hence, we do not consider antenna selection beyond this initial comparison.

The following parameters have been used for the Monte Carlo simulations: $r_{c}=1 \mathrm{~km}$, where $r_{c}$ denotes the cell radius, $\beta=4, \Omega_{s}=1, m_{s}=1$ (which corresponds to a lognormal 
shadowing standard deviation of approximately $7.5 \mathrm{~dB}$ [20]), and $K=3$. There are seven DAPs in the cell, six of which are located uniformly at a distance of $\frac{2}{3} r_{c}$ from the BS, while the seventh DAP is collocated with the central processor at the BS (see Fig. 1). The UTs are randomly dropped in the cell with uniform distribution during each iteration.

Figure 2(a) shows the ergodic aggregate cell spectral efficiency per RB achieved by BD and ZF-DPC in both the CAS and DAS architectures versus $\rho$, where $\rho=P_{t} / \sigma_{n}^{2}$, and Fig. 2(b) shows plots of the outage probability versus the aggregate cell spectral efficiency per RB for each of these schemes at $\rho=20 \mathrm{~dB}$. In this scenario, $\left|\mathcal{C}_{k}\right|=3$ for all $k, N=2$, and $M=2$. The performance gains of the cellular DAS are apparent as both DAS schemes achieve higher aggregate spectral efficiencies than their CAS counterparts. More specifically, at $\rho=20 \mathrm{~dB}$ in Fig. 2(a), each DAS scheme achieves over $60 \%$ higher ergodic aggregate cell spectral efficiency per $\mathrm{RB}$ than the corresponding CAS scheme. Moreover, DAS ZFDPC outperforms DAS BD by approximately 20\%. It is also apparent that both antenna selection and DAP selection for each DAS scheme result in almost the same performance.

Figure 3 shows plots that are similar to those in Fig. 2 with the same parameters, except that now $M=3$. It can be observed that a single additional antenna at each DAP results in improved performance for both DAS schemes. The gap between both DAS schemes is also reduced with DAS ZF-DPC only achieving approximately 6\% higher ergodic aggregate cell spectral efficiency than DAS BD at $\rho=20 \mathrm{~dB}$. In fact, as shown in Fig. 4, adding more antennas at each DAP leads to even better performance, which can be attributed to the availability of excess spatial degrees of freedom since the DAS dimensionality constraint is more than barely satisfied beyond $M=2$. However, this improvement diminishes as the number of antennas per DAP becomes large.

\section{CONCLUSION}

We have extended the BD and ZF-DPC transmission schemes to fit a single-cell DAS architecture with multiantenna DAPs and multi-antenna UTs, and incorporated port selection into these schemes. Each of the two schemes allows multiple UTs to be served in a single RB. Both schemes are simulated for the DAS and CAS architectures under the same total power constraint, which has been imposed for fair comparison. Using the aggregate cell spectral efficiency per $\mathrm{RB}$ as the performance metric, we have shown through simulations that the proposed cellular DAS schemes outperform the corresponding CAS schemes. It has also been shown that port selection, although more restrictive, achieves almost the same performance as the more flexible antenna selection scheme. Furthermore, our simulation results indicate that deploying a greater number of antennas per DAP leads to improved performance, but these performance gains diminish as the number of antennas per DAP becomes large. Although DAS has higher initial deployment costs and additional synchronization complexity as compared to a CAS, the performance improvement that it offers motivates its deployment in future cellular networks.

\section{ACKNOWLEDGMENT}

This work was supported in part by the Ontario Graduate Scholarship (OGS), and CGS M award from the Natural Sciences and Engineering Research Council (NSERC) of Canada.

\section{REFERENCES}

[1] A. Saleh, A. Rustako, and R. Roman, "Distributed antennas for indoor radio communications," IEEE Trans. Commun., vol. 35, no. 12, pp. 1245-1251, Dec. 1987.

[2] H. Yanikomeroglu and E. Sousa, "CDMA distributed antenna system for indoor wireless communications," in Proc. IEEE Int. Conf. on Universal Personal Commun., vol. 2, Oct. 1993, pp. 990-994.

[3] W. Roh and A. Paulraj, "Outage performance of the distributed antenna systems in a composite fading channel," in Proc. IEEE 56th Veh. Technol. Conf., vol. 3, Sep. 2002, pp. 1520-1524.

[4] H. Yanikomeroglu and E. Sousa, "CDMA sectorized distributed antenna system," in Proc. IEEE 5th Int. Symp. Spread Spectrum Techniques and Applications, vol. 3, Sep. 1998, pp. 792-797.

[5] L. Dai, S. Zhou, and Y. Yao, "Capacity analysis in CDMA distributed antenna systems," IEEE Trans. Wireless Commun., vol. 4, no. 6, pp. 2613-2620, Nov. 2005.

[6] W. Roh and A. Paulraj, "MIMO channel capacity for the distributed antenna," in Proc. IEEE 56th Veh. Technol. Conf., vol. 2, Sep. 2002, pp. 706-709.

[7] L. Xiao, L. Dai, H. Zhuang, S. Zhou, and Y. Yao, "Information-theoretic capacity analysis in MIMO distributed antenna systems," in Proc. IEEE 57th Veh. Technol. Conf., vol. 1, Apr. 2003, pp. 779-782.

[8] J. Park, E. Song, and W. Sung, "Capacity analysis for distributed antenna systems using cooperative transmission schemes in fading channels," IEEE Trans. Wireless Commun., vol. 8, no. 2, pp. 586-592, Feb. 2009.

[9] M. Costa, "Writing on dirty paper," IEEE Trans. Inf. Theory, vol. 29, no. 3, pp. 439-441, May 1983.

[10] G. Caire and S. Shamai, "On the achievable throughput of a multiantenna gaussian broadcast channel," IEEE Trans. Inf. Theory, vol. 49, no. 7, pp. 1691-1706, Jul. 2003.

[11] M. Karakayali, G. Foschini, R. Valenzuela, and R. Yates, "On the maximum common rate achievable in a coordinated network," in Proc. IEEE Int. Conf. Commun., vol. 9, Jun. 2006, pp. 4333-4338.

[12] A. Dabbagh and D. Love, "Precoding for multiple antenna gaussian broadcast channels with successive zero-forcing," IEEE Trans. Signal Process., vol. 55, no. 7, pp. 3837-3850, Jul. 2007.

[13] M. Maddah-Ali, M. Sadrabadi, and A. Khandani, "Broadcast in MIMO systems based on a generalized QR decomposition: Signaling and performance analysis," IEEE Trans. Inf. Theory, vol. 54, no. 3, pp. 1124 1138, Mar. 2008.

[14] L. Sun and M. McKay, "Eigenmode transmission for the MIMO broadcast channel with semi-orthogonal user selection," in Proc. IEEE Globecom, Nov. 2009, pp. 2212-2217.

[15] Q. Spencer, A. Swindlehurst, and M. Haardt, "Zero-forcing methods for downlink spatial multiplexing in multiuser MIMO channels," IEEE Trans. Signal Process., vol. 52, no. 2, pp. 461-471, Feb. 2004.

[16] W. Yu and J. Cioffi, "Trellis precoding for the broadcast channel," in Proc. IEEE Globecom, vol. 2, Nov. 2001, pp. 1344-1348.

[17] T. Wang, Y. Wang, K. Sun, and Z. Chen, "On the performance of downlink transmission for distributed antenna systems with multiantenna arrays," in Proc. IEEE 70th Veh. Technol. Conf., Sep. 2009.

[18] L. Ling, T. Wang, Y. Wang, and C. Shi, "Schemes of power allocation and antenna port selection in OFDM distributed antenna systems," in Proc. IEEE 72nd Veh. Technol. Conf., Sep. 2010.

[19] R. Zhang, "Cooperative multi-cell block diagonalization with per-basestation power constraints," IEEE J. Sel. Areas Commun., vol. 28, no. 9, pp. 1435-1445, Dec. 2010.

[20] I. Kostic, "Analytical approach to performance analysis for channel subject to shadowing and fading," IEE Proc.-Commun., vol. 152, no. 6 , pp. 821-827, Dec. 2005. 


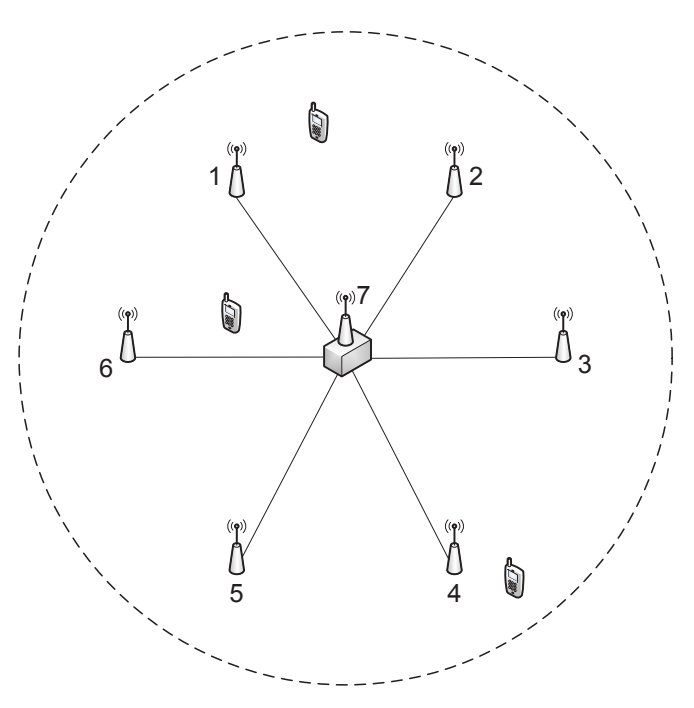

Fig. 1. A single-cell DAS with $K=3$ and $D=7$.
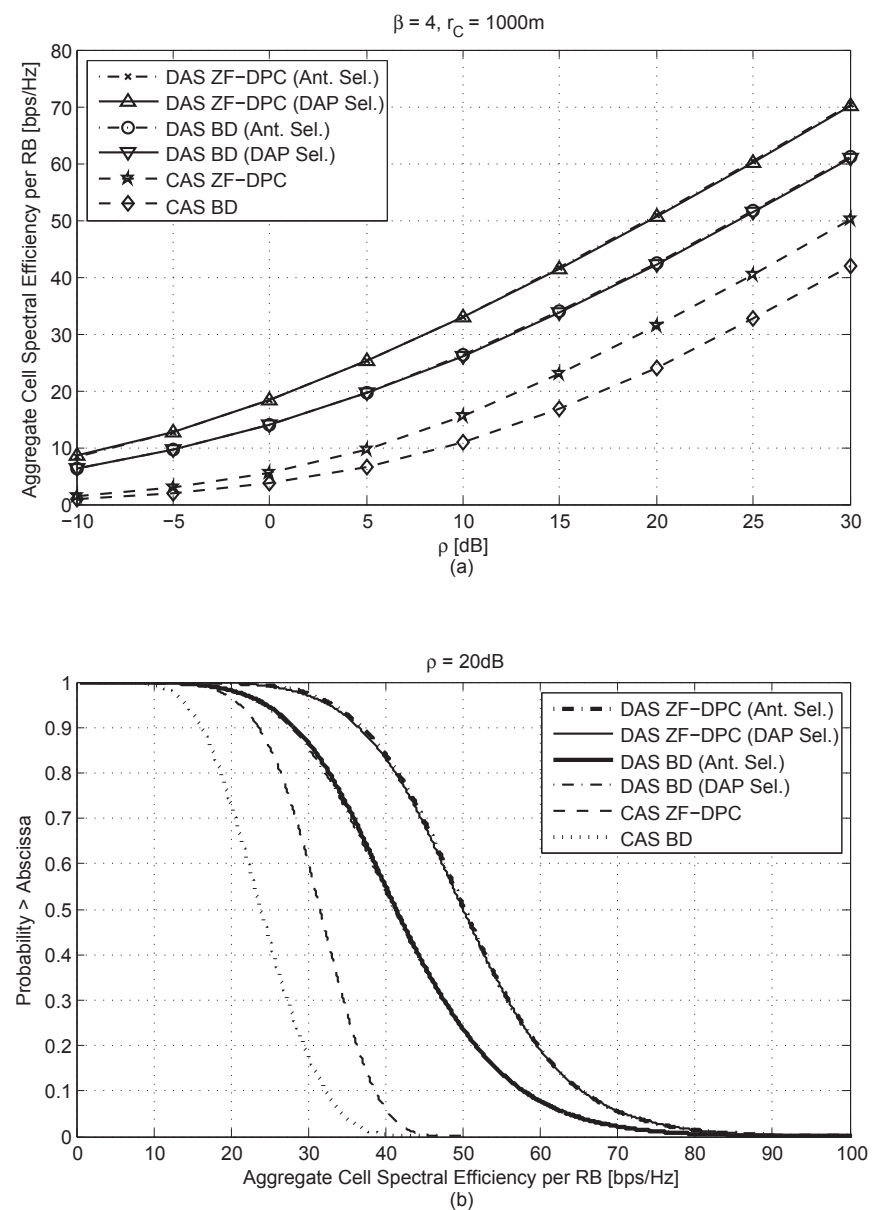

Fig. 2. Comparison of ergodic and outage aggregate cell spectral efficiency per RB for BD and ZF-DPC under the CAS and DAS architectures with $K=3, N=2, M=2$, and $\left|\mathcal{C}_{k}\right|=3, \forall k$.
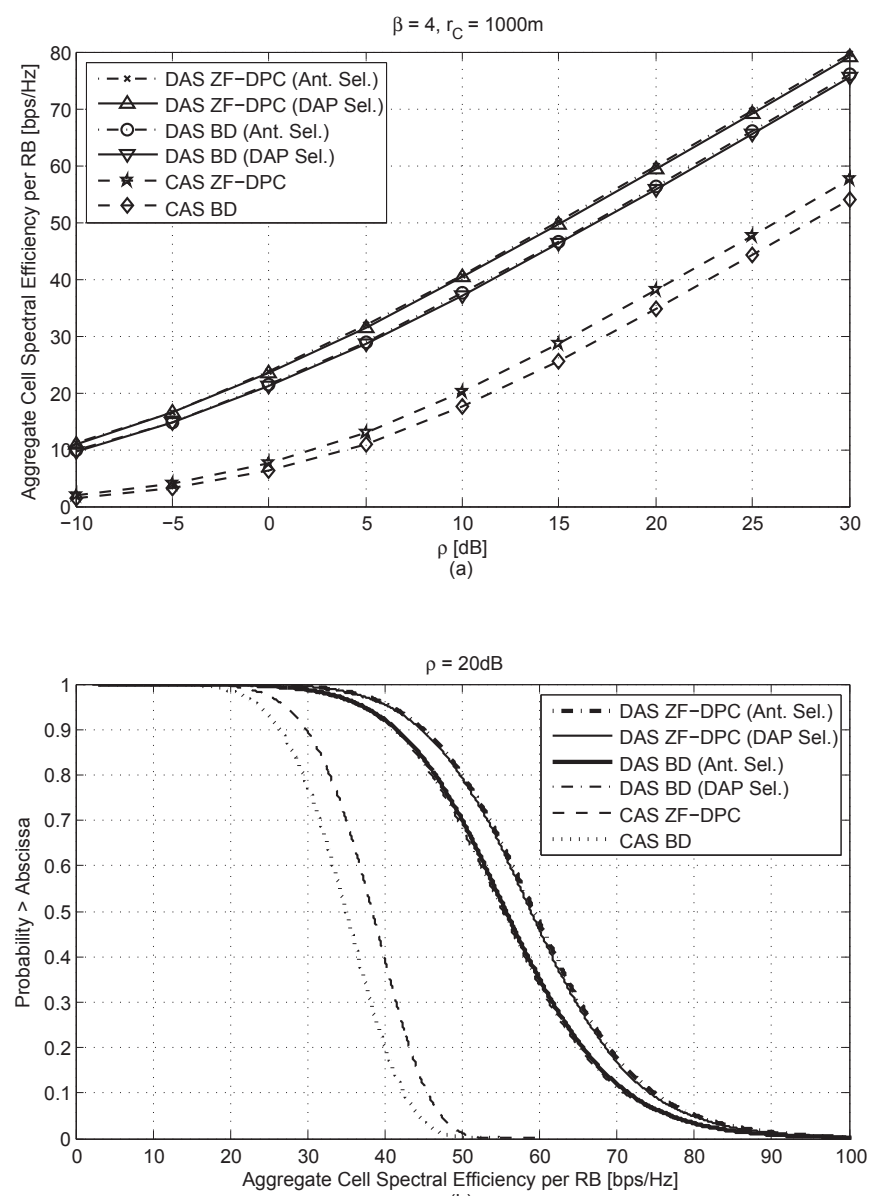

(b)

Fig. 3. Comparison of ergodic and outage aggregate cell spectral efficiency per RB for BD and ZF-DPC under the CAS and DAS architectures with $K=3, N=2, M=3$, and $\left|\mathcal{C}_{k}\right|=3, \forall k$.



Fig. 4. Ergodic aggregate cell spectral efficiency per RB for BD and ZF-DPC under the CAS and DAS architectures for different numbers of antennas per DAP with $K=3, N=2$, and $\left|\mathcal{C}_{k}\right|=3, \forall k$. 